\title{
Fundamentals of Metal Oxide Gas Sensors
}

\author{
Nicolae Barsan and Udo Weimar \\ Institute of Physical and Theoretical Chemistry, University of Tuebingen, Auf der Morgenstelle 15 \\ 72076 Tuebingen, Germany
}

\begin{abstract}
:
The contribution presents the results of "operando" investigations and theoretical modeling of gas sensing with semiconducting metal oxides based sensors. It discusses the role of the different parts of the sensors and demonstrates that the measured effects are determined by their interaction.
\end{abstract}

Key words: SMOX gas sensors, "operando" investigations, conduction mechanisms, water vapor cross-interference, CO detection.

\section{Introduction}

Chemoresistive gas sensors based on semiconducting metal oxides (SMOX) are sold in millions for solving applications spanning from the detection of explosive gas leakages in residential premises to hindering the access of pollutants inside cars. All started after metal oxides were identified as possible sensitive materials by Heiland et al. [1], Bielanski et al. [2] and Seiyama et al. [3] and brought to the market by Taguchi [4](1971), who founded the still largest manufacturer of SMOX sensors, namely Figaro Engineering (Figaro). They were so successful because they are inexpensive, easy to use, very sensitive and stable, easy to integrate into arrays and low power when realized on the appropriate transducer structures.

In principle, gas detection with SMOX based gas sensors is simple: in air, at temperatures between 150 and $400^{\circ} \mathrm{C}$, oxygen is adsorbed at the surface of the metal oxides by trapping electrons from the bulk with the overall effect of increasing the resistance of the sensor, for $n$ type materials, or decreasing it, for p-type materials. The occurrence of a target gas in the atmosphere, which reacts with the preadsorbed oxygen or directly with the oxide, determines a change of the sensor resistance, which is recorded as a sensor signal and the magnitude of which is correlated to the concentration of the target gas. Even from this simplified picture it is clear that one has to examine two aspects: the surface reaction taking place between the material and the gases (called the receptor function) and the transduction of it into the corresponding changes of the electrical resistance of the sensor. In this contribution we will examine more in detail the way in which the actual SMOX based gas sensors function, taking into consideration all their relevant parts, and then will use the example of $\mathrm{CO}$ detection to illustrate the power of "operando" investigation techniques to explain the dependence of sensor signals on ambient conditions.

\section{Overview of Sensing}

Fig. 1 shows one electron microscope picture of an actual sensing layer of a state of the art SMOX gas sensor; it is based on $\mathrm{SnO}_{2}$ and it is realized by screen printing the sensing material onto an $\mathrm{Al}_{2} \mathrm{O}_{3}$ substrate provided with $\mathrm{Pt}$ electrodes.

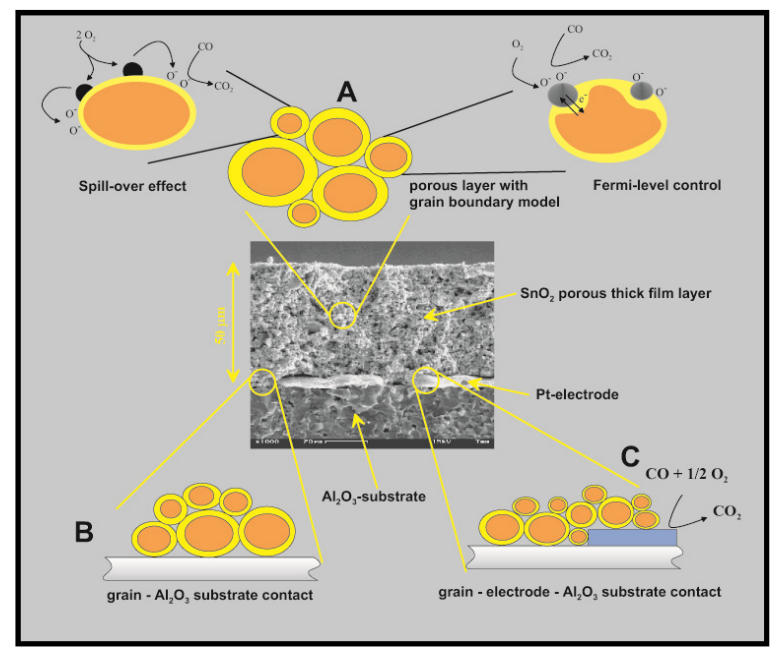

Fig. 1. Illustration of sensor parts with possible impact on the sensing properties. 
In the same figure cartoon like illustrations of the parts that could influence the sensing properties are provided (for a detailed analysis, see [5]). They are:

1. The $\mathrm{SnO}_{2}$ grains at the surface of the layer of which the reactions with the ambient gases are taking place and between which the transport of the free charge carriers takes place. In the figure the lighter color at the surface of the grains indicates the depletion region determined by the ionosorption of oxygen.

2. Different material synthesis methods will result in different bulk and surface properties, which both have an influence either through different surface reactivity/receptor function and/or through the conduction mechanism - on the sensors' performance.

3. At the surface of the $\mathrm{SnO}_{2}$ grains noble metal additives can be/are present. In the figure some possible sensitization mechanism are illustrated.
4. The noble nature of the electrodes makes it possible for them to play an active chemical role. This means that their nature but also micro-structure and morphology will have an impact on gas sensing performance.

As a result one can say that the sensor is more than the "algebraic" sum of its parts and in order to understand the way in which the different parts influence the whole one needs investigation techniques that will allow gaining a deep insight. Moreover, because of the very well-known differences between the properties of ideal, in our case single crystal, and real, polycrystalline, surfaces we need to apply all the techniques in conditions as close as possible to the real operation ones.

\section{“Operando" Investigations}

In order to be able to meet these requirements in the last 15 years we developed such an approach; an overview of the different techniques is presented in Fig. 2 (for details, see [6]). They are providing complementary information and are allowing getting unprecedented insight into gas sensing.

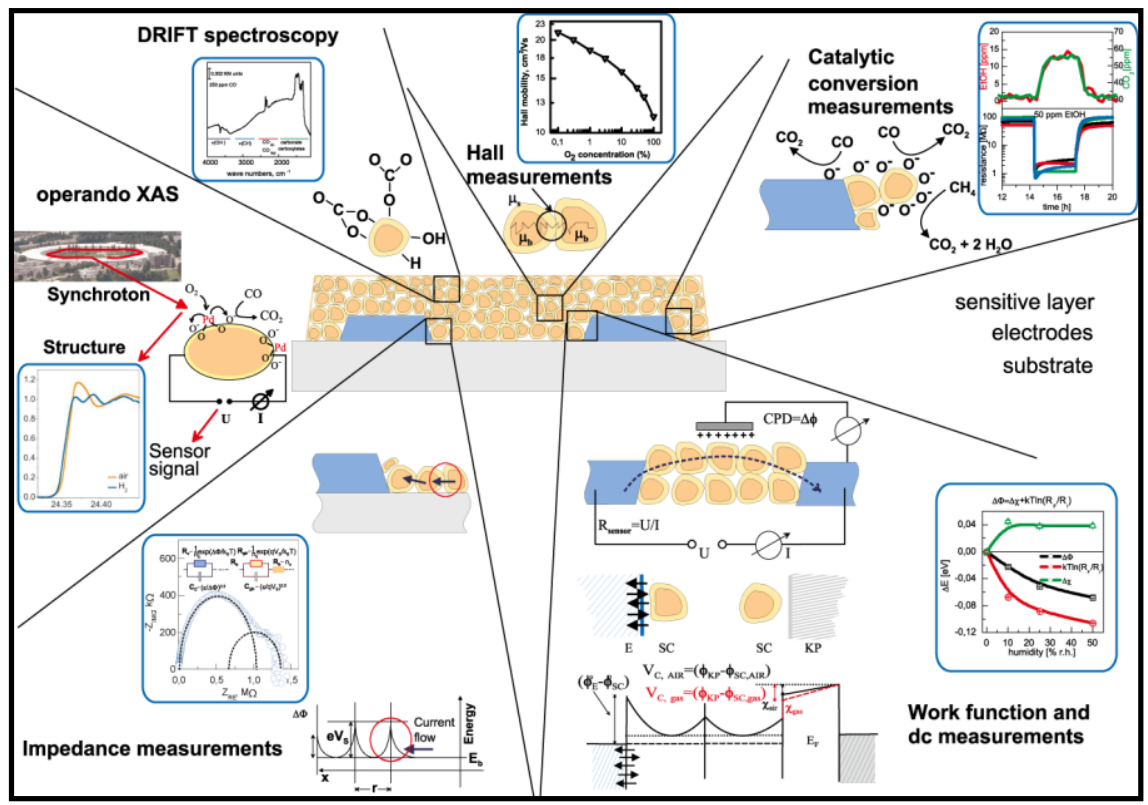

Fig. 2. Overview of "Operando" investigation techniques.

The techniques are:

- DC resistance measurements (the normal way to characterize gas sensors) give information about the changes in the concentration of free charge carriers induced by surface reactions.

- AC impedance spectroscopy allows identifying the presence of surface space charge layers or electrode-metal oxide contacts and of the nature of free charge carriers (ions or electrons). In combination with work function changes measurements it permits to follow the changes in the surface dipole concentration. Some examples are provided in [7] and [8].

- Hall Effect measurements in combination with the modeling of the conduction will allow to deconvolute the 2 standard components: the charge carrier concentration and their drift mobility of the 
conductivity. It will also provide information about the sign of the majority charge carriers. An example is provided in [9].

- Work function change measurements by the Kelvin method provide insight about relative changes in the work function determined by surface reactions (charge transfer processes between gases and metal oxides). In combination with conductance measurements it allows for the discrimination between localized chemisorption and ionosorption, e.g. effects related to dipoles and ions, and also for the investigation of surface and bulk properties of the gas sensitive SMOX (see, e.g. [7] and [10]).

- On-line gas analysis (paramagnetic oxygen analyzer, IR gas monitors ( $\mathrm{CO}, \mathrm{CO}_{2}$ etc.), chemiluminescence $\mathrm{NO}_{x}$ analyser, IR ozone analyzer, on-line MS) of exhaust composition after the sensor reactor allows to identify reaction products of solid-gas interaction and yields information about possible reaction mechanisms (an example in [11]).

- Diffuse Reflection Infrared Fourier Transformed Spectroscopy (DRIFTS) measurements identify adsorbed surface species which are involved in the gas solid reaction; it is one of the few spectroscopic techniques possible to be applied in"operando" and its input is essential for the identification of reaction mechanisms (an example in [12]).

- X-ray absorption spectroscopy studies, which allow finding out the chemical state, the surrounding and possible changes in the chemical state of, e.g., noble metal additives used as sensitizers in SMOX gas sensors (an example in [13]).

By using the insight provided by some of those investigation methods, the mechanism of water vapor interference in the detection of $\mathrm{CO}$ was clarified.

\section{CO Sensing with $\mathrm{SnO}_{2}$ based Gas Sensors}

$\mathrm{CO}$ is a very important target gas because of its toxicity, which combined with its lack of odor, makes it very dangerous in households using ovens for heating and in a series of industrial processes in which one encounters incomplete combustion processes. Water vapor is the most relevant interfering gas in environmental applications. Because the chemistry of $\mathrm{CO}$ detection is rather simple, involving the oxidation of $\mathrm{CO}$ to $\mathrm{CO}_{2}$, this type of detection task is "relatively" simple so it can be used as a model case for the understanding of the involved mechanisms.
In the case of undoped $\mathrm{SnO}_{2}$, our findings are indicating the following:

- Ambient oxygen is ionosorbed at the surface of the metal oxide, by that negatively charging it, which determines the upwards energy band bending at the surface and the increase of the sensor resistance. The work function changes measurements are proving that the only effect of oxygen adsorption is the upwards bending of the energy bands;

- Ambient water vapor reacts with the preadsorbed oxygen ions forming surface hydroxyl groups. The decrease of the concentration of oxygen ions is proven by the decrease of the sensor resistance and by the decrease of band bending in work function and resistances changes measurements. The formation of the hydroxyl groups is proven by DRIFTS experiments and by the increase of the electron affinity recorded in work function changes measurements;

- Exposure to CO determines a competition with the water molecules for pre-adsorbed oxygen ions. The effect is a decrease of the sensor signal when compared to the situation encountered in dry air but also a decrease of the concentration of the surface hydroxyl groups. The latter is demonstrated by the: observed decrease of the corresponding IR adsorption bands during DRIFTS experiments; the decrease of the electron affinity recorded in the work function changes experiments.

It is very important that all those steps are experimentally proven by a combination of phenomenological and spectroscopic investigation techniques that are performed in the same conditions and on samples which are having all characteristics of real sensors. Also, the experimental conditions are very close to the ones the sensors will face in real applications: normal pressure, humid air and at normal operation temperatures (around $300^{\circ} \mathrm{C}$ ).

Fig. 3 illustrates the chemical processes - the reaction of $\mathrm{CO}$ with the ionosorbed oxygen ion to $\mathrm{CO}_{2}$ - and their impact on the surface band bending and electron affinity.

Besides the example of $\mathrm{CO}$ sensing with undoped $\mathrm{SnO}_{2}$, additional results will be presented on the following topics: effects of the nature of electrodes; impact on bulk and surface properties of the additives; impact of $\mathrm{Pt}$ and $\mathrm{Pd}$ doping on the water vapor cross interference. Also the impact of conduction mechanisms on the sensing performance will be discussed. 


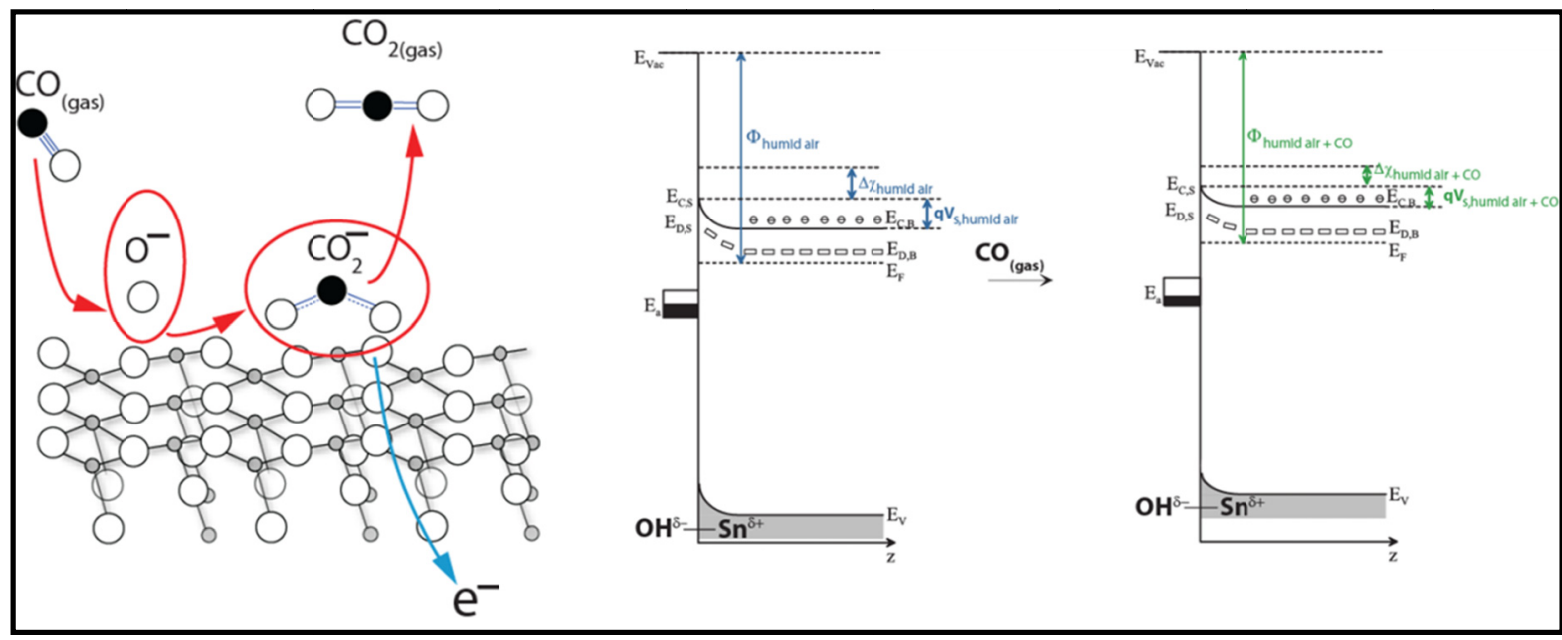

Fig. 3. Cartoon and energy bands representation of processes taking place during CO detection in humid air.

\section{Conclusions}

The processes involved in gas sensing with SMOX based gas sensors are extremely complex and their understanding, which is needed for being able to develop in an intelligent manner better sensors, asks for very performing investigation techniques that are to be applied in operation conditions.

\section{Acknowledgements}

The authors are thankful to the German Research Foundation (DFG) for the financial support provided through the SPP 1299 - „Das Haut-Konzept", WE 3662/1-2 and MA 3333/2-1.

\section{References}

List and number all bibliographical references at the end of the paper. When referenced within the text, enclose the citation number in square brackets, i.e. [1]

[1] G. Heiland, Zum Einfluss von Wasserstoff auf die elektrische Leitfähigkeit von ZnO-Kristallen, Z.Physik, 138, 459-464 (1954); doi: 10.1007/BF01327362

[2] A. Bielanski, J. Deren and J. Haber, Electric conductivity and catalytic activity of semiconducting oxide catalysts, Nature, 179, 668-669 (1957); doi: 10.1038/179668a0

[3] T. Seiyama, A. Kato, K. Fujiishi and M. Nagatani, A new detector for gaseous components using semiconductive thin films, Anal Chem, 34, 1502f (1962); doi: 10.1021/ac60191a001

[4] N. Taguchi (1971) US Patent No. 3631436.

[5] N. Barsan and U. Weimar, Conduction Model of Metal Oxide Gas Sensors, Journal of Electroceramics 7, 3 (2001) 143-167; doi: 10.1023/A:1014405811371

[6] N. Barsan, D. Koziej and U. Weimar, Metal oxide based gas sensor research: how to? Sensors and Actuators B 121 (2007) 18-35; doi: 10.1016/j.snb.2006.09.047
[7] N. Barsan and U. Weimar, Understanding the fundamental principles of metal oxide based gas sensors: the example of $\mathrm{CO}$ sensing with $\mathrm{SnO}_{2}$ sensors in the presence of humidity, J. Phys.: Condens. Matter 15 (2003) R1-R27; doi: PII S0953-8984(03)33587-8

[8] Suman Pokhrel, C.E. Simion, V. Quemener, N. Bârsan, U. Weimar, Investigations of conduction mechanism in $\mathrm{Cr}_{2} \mathrm{O}_{3}$ gas sensing thick films by ac impedance spectroscopy and work function changes measurements, Sensors and Actuators B 133, 1 (20108) 78-83; doi: 10.1016/j.snb.2008.01.054

[9] A. Oprea, E. Moretton, N. Bârsan, W. J. Becker, J. Wöllenstein, U. Weimar, Conduction model of $\mathrm{SnO}_{2}$ thin films based on conductance and Hall effect measurements, Journal of Applied Physics 100, 3 (2006) Article Number: 033716; doi: $10.1063 / 1.2229802$

[10] M. Hübner, N. Bârsan, U. Weimar, Influences of $\mathrm{Al}, \mathrm{Pd}$ and $\mathrm{Pt}$ additives on the conduction mechanism as well as the surface and bulk properties of $\mathrm{SnO}_{2}$ based polycrystalline thick film based gas sensors, accepted for publication in Sensors and Actuators B

[11] M. Hübner, C. E. Simion, A. Haensch, N. Barsan and $\mathrm{U}$. Weimar, $\mathrm{CO}$ sensing mechanism with $\mathrm{WO}_{3}$ based gas sensors, Sensors and Actuators B 151, 1 (2010) 103-106; doi: 10.1016/j.snb.2010.09.040

[12] D. Koziej, K. Thomas, N. Barsan N, F. ThibaultStarzyk, U. Weimar, Influence of annealing temperature on the $\mathrm{CO}$ sensing mechanism for tin dioxide based sensors-Operando studies, Catalysis Today 126 1-2 (2007) 211-218; doi: 10.1016/j.cattod.2007.03.011

[13] M. Huebner, D. Koziej, M. Bauer, N. Barsan, K. Kvashnina, M. D. Rossell, U. Weimar, J. D. Grunwaldt, The Structure and Behavior of Platinum in $\mathrm{SnO}_{2}$-Based Sensors under Working Conditions, Angewandte Chemie-International Edition 50, 12 (2011) 2841-2844; doi: 10.1002/anie.201004499 